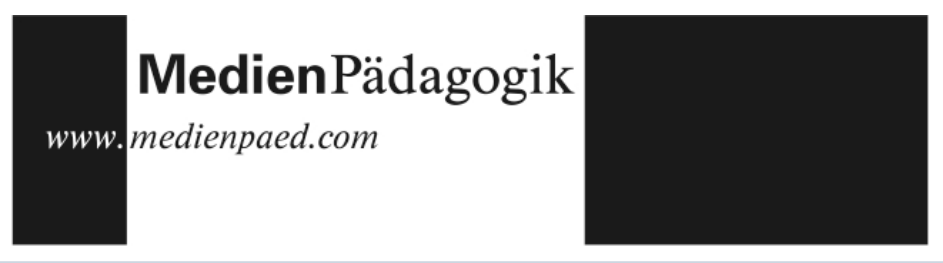

Rezensionen

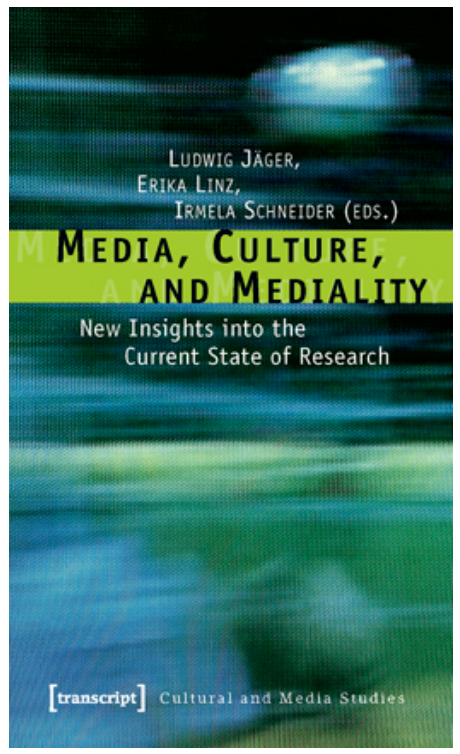

Ludwig Jäger, Erika Linz, Irmela Schneider (Eds.)

Media, Culture, and Mediality

New Insights into the Current State of Research

Bielefeld: transcript. 2010. 464 Seiten

ISBN: 978-3-8376-1376-6

$€ 39,80 ;$ CHF 48,60

\title{
Medien, Kommunikation und Kultur im Zusammenspiel
}

Von Januar 1999 bis Ende 2008 bestand mit Unterstützung der Deutschen Forschungsgemeinschaft an den Universitäten Aachen, Bonn und Köln ein interdisziplinärer Forschungsschwerpunkt zum Thema «Media and Cultural Communication»; Vorarbeiten und Vorbereitungen reichen bis in die Anfänge der 1990er Jahre zurück. Dieser umfangreiche Sammelband mit 21 Beiträgen versteht sich wohl als Abschlussdokument dieses gemeinsamen interdisziplinären Vorhabens, wobei nicht alle Beteiligten und Beiträger, wie die Herausgeber/innen in ihrer Einleitung einräumen, aus Platzgründen vertreten sein konnten. Übergreifend sind kultur- und medienwissenschaftliche Sichtweisen, vielfach kombiniert mit historischen und rekonstruierenden Zugängen, um - wie es heißt - das Zusammenspiel zwischen Medien, Kultur und Kommunikation auf vielfältige Weise analytisch zu ergründen und theoretisch abzubilden. Dabei wird durchaus berücksichtigt, gewissermaßen als forschungs- und erkenntnisleitende Prämissen, dass zum einen Kultur seit den 1970er Jahren mehr und mehr medial konstituiert und durchdrungen wird, zunächst von den audiovisuellen Massenmedien, inzwischen auch von den digitalen Formaten. Zum anderen muss damit impliziert werden, dass technologische Konditionen weithin kulturelle Wahrnehmungen und Erfahrungen beeinflussen, wenn nicht prägen. Und endlich muss angenommen werden - wohl in Anlehnung an die Systemtheorie (was allerdings nicht generell expliziert wird) -, dass Medien eine maßgebliches soziales Subsystem darstellen, ja für moderne Gesellschaften und deren Funktionieren unverzichtbar sind. 
Aus diesen Prämissen haben sich drei Forschungsfelder in vielfältigen Konzeptionen und Diskussionen herausgeschält, die natürlich aufeinander Bezug nehmen und sich hier in drei Teilen abbilden: erstens das Konstrukt der Medialität in vielerlei Varianten und Nuancen, zunächst, in der Phase der Massenkommunikation, eher als Ausdifferenzierung einzelner Medien, Gattungen und Formate wirksam, inzwischen, mit der anhaltenden Digitalisierung, eher als wachsende Konvergenz und Hybridisierung, was hier allerdings nicht ausführlich betrachtet und dargestellt wird. Für «Konzepte und Modelle» von Medialität stehen in diesem Reader sechs Beiträge, die recht allgemeine (z.B. Beschreibungen und Typologien von Medien, Medium als Form), aber ebenso spezielle Aspekte (z.B. Nachrichtenwerttheorien) bearbeiten sowie systematische und historische Zugänge (z.B. Begrifflichkeiten und theoretische Entwürfe, vom Kommunikationskanal bis zur Medienbotschaft von 1945 bis 1960) aufgreifen.

Das zweite Forschungsterrain erstreckt sich auf Medien-Diskurse als analytische Betrachtungen von Medien, genauer von Dispositiven und Politiken von Medien. Sie sind dabei in funktionaler Hinsicht doppelt präsent: als Faktoren zur Initiierung, Beschleunigung, aber ebenso zur Retardierung des Wandels wie auch als diskursiver Reflex und plastische Selbstthematisierung dessen, was passiert. Auf breiter, vielschichtiger Materialbasis werden dafür Anschauungsbeispiele analysiert. Sie finden sich in sieben Beiträgen, die wiederum breit gefächert sind: Von den schockierenden Bildern aus dem irakischen Abu Ghraib, über die Rhetorik Hitlers bis hin zu politischen Strategien zum Fernsehen als 〈Problem» und als «Instrument».

Der dritte Forschungsbereich dürfte der heterogenste, aber auch der sein, der den herkömmlichen Philologien und Kulturwissenschaften am nächsten geblieben ist. Er kümmerte sich um die Entwicklungen so genannter Kommunikationskulturen, noch pragmatischer darum: wie Kommunikation unter den gegenwärtigen Kommunikationsbedingungen möglich und erfolgreich sei. Überschrieben ist er in dem Reader mit «Ästhetiken und Modalitäten». Entsprechend disparat befassen sich die acht Beiträge etwa mit elementaren Aspekten persönlicher Kommunikation, mit Medien- und Bildtheorien von W. Benjamin, J. C. Lavater und G. C. Lichtenberg, mit den Reaktionsexperimenten von I. P. Pavlov Anfang des 20. Jahrhunderts sowie mit der multimedialen Popliteratur von R. D. Brinkmann und anderen. 
Wenngleich die Herausgeber/innen die intensiven und fortgesetzten Diskussionen in dem Forschungsschwerpunkt loben, die zu vielen transdisziplinären Ideen, Konzepte und Methoden führten und so die Untersuchungsbereiche unter vielfältigen Vorzeichen und Zugängen sondieren ließen (dem Band sogar eine Art kollektiver Autorenschaft bescheinigen), erschließen sich für den Leser die Korrespondenzen und Befruchtungen zwischen den Artikeln nur streckenweise und mit Mühen. Ein auch nur annähernd theoretisches Konzept dessen, was eingangs als komplexes Zusammenspiel und vielschichtige Interferenzialität von Medien, Kommunikation und Kultur apostrophiert worden ist (und wofür es ja in diversen Disziplinen vielerlei Ansätze und Bestrebungen gibt), kommt in diesem Sammelband allenfalls sporadisch und fakultativ zum Vorschein.

Hans-Dieter Kübler 